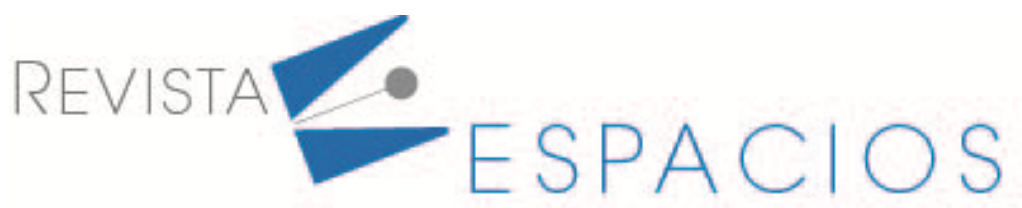

\title{
Los distritos industriales zapateros de las comarcas españolas del Vinalopó: Un análisis detallado
}

\section{The footwear industrial districts of the Spanish Vinalopo regions: A detailed analysis}

\author{
MARCO LAJARA, Bartolomé ${ }^{1}$ \\ ZARAGOZA SAEZ, Patrocinio C. ${ }^{2}$ \\ MARTÍNEZ FALCÓ, Javier ${ }^{3}$ \\ SEVA LARROSA, Pedro ${ }^{4}$
}

\begin{abstract}
Resumen
La presente investigación tiene como objetivo contrastar si los distritos industriales zapateros de las comarcas españolas del Vinalopó siguen teniendo vigencia en la actualidad, o si, por el contrario, la actividad zapatera desarrollada en dichas aglomeraciones empresariales ha perdido relevancia en favor de otras actividades económicas. Además, se analiza cómo dichos distritos actúan como polo de atracción de la inversión extranjera.
\end{abstract}

Palabras clave: distrito industrial, calzado, vinalopó, polos de atracción.

\begin{abstract}
In this research, it is contrasted whether the footwear industrial districts of the Spanish regions of Vinalopo are still valid today, or if, on the contrary, the shoemaking activity carried out in these business agglomerations has lost relevance in favor of other economic activities. In addition, it is analyzed how these districts act as a pole of attraction for foreign investment.

Key words: industrial district, footwear, vinalopo, poles of attraction.
\end{abstract}

\section{Introducción}

En la literatura económica se han derramado ríos de tinta acerca de las ventajas obtenidas por las grandes empresas, las cuales pueden generar economías de escala y de experiencia, obtener superioridades tecnológicas, así como mayores recursos que pueden redundar en mejores resultados. Sin embargo, las Pequeñas y Medianas Empresas (PYMES), que al 1 de enero de 2019 representaban el 99,99\% de las empresas españolas según datos extraídos del Directorio Central de Empresas (DIRCE), poseen también un conjunto de ventajas que se derivan principalmente de la flexibilidad y la rapidez en la toma de decisiones que les permite su tamaño.

Las aglomeraciones territoriales de empresas ayudan a que estas últimas puedan obtener numerosas ventajas (de escala, de localización, etc.) a través de la cooperación y la colaboración entre ellas. En esta línea de pensamiento, los distritos industriales, definidos como "una entidad socioterritorial que se caracteriza por la

\footnotetext{
${ }^{1}$ Catedrático de Universidad. Departamento de Organización de Empresas. Universidad de Alicante (España). Correo electrónico: bartolome.marco@ua.es 2 Profesora Titular de Universidad. Departamento de Organización de Empresas. Universidad de Alicante (España). Correo electrónico: patrocinio.zaragoza@ua.es

${ }^{3}$ Profesor Ayudante. Departamento de Organización de Empresas. Universidad de Alicante (España). Correo electrónico: javier.falco@ua.es

${ }^{4}$ Profesor Ayudante Doctor. Departamento de Organización de Empresas. Universidad de Alicante (España). Correo electrónico: pedro.seva@ua.es
} 
presencia activa tanto de una comunidad de personas como de un conjunto de empresas en una zona natural e históricamente determinada" (Becattini, 1990), han generado un gran interés en los estudios de Dirección Estratégica. Los distritos industriales influyen en la competitividad de las empresas localizadas en los mismos, puesto que éstos ofrecen una serie de externalidades positivas que permiten obtener resultados operativos excelentes sin la necesidad de obtener los recursos de las grandes empresas.

Asimismo, estudios como el de Boix y Galleto (2006a) y Boix y Trullén (2010) ponen en valor la importancia de los distritos industriales en España, destacando la existencia de concentraciones empresariales de este tipo en trece de las diecisiete regiones españolas, con predominio de las concentraciones en la Comunidad Valenciana. En los estudios también se destaca la figura del distrito por concentrar un gran porcentaje de las exportaciones nacionales y por su capacidad innovadora, al ser superior a la media nacional, lo que corrobora el papel fundamental de los distritos industriales en la senda innovadora del país (Boix y Galleto, 2006b).

En la presente investigación se han analizado los distritos industriales españoles del calzado localizados en las comarcas del Vinalopó, por lo que dichas comarcas alicantinas constituyen nuestra unidad de análisis. La decisión de analizar las comarcas del Vinalopó no ha sido azarosa, pues es ahí donde existe una gran concentración espacial de empresas dedicadas a la industria del calzado, concentrando alrededor del $60 \%$ de la producción nacional. Además, se produce una distribución de especialidades en dichas comarcas (Sebastiá, 1997). De este modo, la tradición del calzado en cáñamo y esparto influye en que en Elche y su comarca -el Bajo Vinalopó- se localice el calzado realizado con gran cantidad de caucho y plástico. Por otro lado, la zona de Elda -el Medio Vinalopó-, se ha especializado en calzado de señora y la de Villena -el Alto Vinalopó-, a partir de la experiencia en el trabajo con el cuero, se ha especializado en el calzado de niño. Las razones de esta especialización productiva se han atribuido, sobre todo, a la presencia de mano de obra especializada, a la tradición que enlaza con la existencia local de una materia prima (como por ejemplo el esparto), al conocimiento del mercado y a la facilidad de acceder a información sobre nuevas materias primas, máquinas, clientes, etc.

Los distritos zapateros españoles de las comarcas del Vinalopó ya han sido identificados (Boix y Galleto, 2004 y 2006a). Sin embargo, los datos empleados para su identificación corresponden al año 2001. En la presente investigación se pretende contrastar si dichos distritos identificados en trabajos previos siguen teniendo vigencia en la actualidad, o si por el contrario, tras casi dos décadas, la actividad principal desarrollada hasta ese momento en dichas concentraciones empresariales ha perdido relevancia en favor de otro tipo de actividades económicas. Además, se pretende analizar cómo los distritos zapateros del Vinalopó actúan como polo de atracción de la inversión extranjera, pues si bien varios autores han hecho aportaciones al proceso de internacionalización de la industria del calzado en España (Belso, 2006; Miranda, 2014) no se ha abordado su estudio desde la perspectiva del distrito industrial como polo de atracción de nuevas empresas que aportan nuevas ideas y ayudan al distrito a evolucionar.

En conclusión, dada la importancia que este tipo de concentraciones empresariales tiene en España, podemos justificar la realización de la presente investigación por los siguientes motivos:

1. La necesidad de seguir estudiando la realidad de los distritos industriales, su funcionamiento y el origen de sus ventajas competitivas.

2. La importancia de las comarcas españolas del Vinalopó como centro neurálgico de la producción del calzado a nivel nacional.

3. La necesidad de contrastar la vigencia de los distritos zapateros del Vinalopó en la actualidad.

4. Analizar cómo los distritos zapateros del Vinalopó actúan como polos de atracción de la inversión extranjera.

La investigación se estructura de la siguiente manera. En primer lugar, tras esta breve introducción, se expone el marco teórico donde se realiza una pequeña aproximación al sector del calzado español y a los distritos industriales. En segundo lugar, se procede a explicar la metodología seguida en la parte empírica con el fin de poder abordar los diferentes objetivos planteados. En tercer lugar, se presentan los principales resultados 
obtenidos en la investigación. Finalmente, en el último apartado se recogen las principales conclusiones obtenidas a partir de la realización del trabajo, exponiendo las aportaciones de la investigación más relevantes.

\section{Marco teórico}

Antes de adentrarnos en el análisis de la metodología y los resultados de la investigación, resulta conveniente realizar una aproximación al sector del calzado español, sus características, su proceso de internacionalización, así como las ventajas que se derivan de los distritos industriales, pues éstos constituyen nuestra unidad de análisis. Según la definición de Cervera (2003) el sector del calzado está integrado por toda una serie de actividades industriales y manufactureras que tienen por objeto la transformación, de forma secuencial, de una serie de materias primas para la obtención de un producto final (zapatos), para que sean comercializados en los distintos puntos de venta.

La mayor parte de las empresas que componen este sector en España son PYMES $(52,09 \%)$ y microempresas $(47,85 \%)$ por lo que es posible afirmar que el sector del calzado está muy atomizado. Además, su ubicación geográfica suele estar concentrada en determinadas comunidades autónomas entre las que destacan la Comunidad Valenciana (66,53\%), Castilla La Mancha $(9,41 \%)$ y La Rioja $(9,13 \%)$.

El proceso de globalización de la economía ha provocado el aumento de la competencia internacional en el sector del calzado en los últimos años, destacando la presencia de países asiáticos entre sus principales competidores. Este hecho ha potenciado la deslocalización de las empresas y el descenso en la producción y las exportaciones. Así, esta industria ha protagonizado cambios profundos como son: la crisis económica que ha afectado a países tradicionalmente importadores del calzado español, la ralentización de la economía, con la subsiguiente disminución de la demanda de este tipo de bienes y la inestabilidad en los mercados de divisas internacionales, con cambios en el volumen de exportaciones y oscilaciones de coste de materias primas.

\subsection{Características del sector}

El sector del calzado español se ha caracterizado históricamente por su dinamismo y pluralidad, pues dentro de él pueden encontrarse diversos modelos y estrategias empresariales. La transformación experimentada en los últimos años ha permitido a la industria mejorar su nivel de competitividad y con ello, mejorar su presencia en los mercados internacionales, logrando cifras elevadas de exportación. A continuación, se enumeran algunas de las características más significativas de la industria española del calzado:

- Gran contribución a la economía española. Los datos del sector revelan una importante cifra de contribución en términos de empleo y producción, sin incluir la actividad productiva que las empresas españolas realizan fuera del país y que son difícilmente cuantificables.

- Dependencia del consumo final. La naturaleza de la industria del calzado está sujeta a la coyuntura económica del país y a las variaciones en la demanda final de éste. Así, en los años de crisis, los sectores asociados a la moda y a las industrias manufactureras en general han sufrido un importante descenso en su demanda.

- Atomización de la estructura empresarial. El volumen de compañías grandes y medianas pertenecientes a la industria del calzado en España es muy reducido (Climent y Méndez, 2002), siendo más abundantes las empresas de pequeño tamaño (con menos de 50 trabajadores) y las micro (con menos de 10 trabajadores).

- Orientación a la exportación y reposicionamiento en mercados exteriores. La caída de la demanda interna ha fomentado la actividad exportadora del sector. El mantenimiento de las empresas del calzado en los mercados internacionales ha sido posible gracias al refuerzo en la competitividad de las mismas. La actividad exportadora se ha realizado mayoritariamente hacia países europeos, con propensiones más ambiciosas como Estados Unidos, China y Rusia entre otras (Fuster, Martínez y Pardo, 2008). 
- Complejidad del negocio. El calzado está caracterizado por ser un sector con una cadena de valor muy fragmentada en su fase productiva, en la que intervienen gran número de actores (Tortajada, Fernández e Ybarra, 2005).

Aumento de la competencia internacional. La globalización general ha influido al sector del calzado, haciendo que su entorno sea cada vez más competitivo como consecuencia de la continua aparición de nuevos entrantes que compiten con precios muy bajos. Como respuesta, el sector español ha optado, generalmente, por estrategias de marca, calidad, diseño y moda con grandes inversiones en innovación (Tortajada, Fernández e Ybarra, 2005).

- Convivencia de varios modelos de negocio. La adaptación al entorno ha fomentado la aparición de modelos de negocio cooperativos o de multilocalización empresarial en el sector.

- Desarrollo tecnológico avanzado. Las innovaciones tecnológicas tienen gran aplicación en la industria del calzado, destacando las actividades de innovación en nuevos materiales, sistemas de logística y distribución, tecnologías avanzadas de diseño y fabricación, etcétera (Ybarra y Santa María, 2005).

\subsection{Fases en la evolución y desarrollo del sector}

De acuerdo con Tortajada, Fernández e Ybarra (2005), las últimas décadas del sector del calzado español están determinadas por tres fases distintas en la evolución y desarrollo del sector. Estas fases han estado influenciadas por cambios significativos tanto en la estructura del sector, como en la tecnología, la innovación, los recursos humanos y la demanda del mercado. Así, podemos señalar las siguientes fases:

Fase I. Esta fase se relaciona con la década de los sesenta hasta mitad de los setenta. El intenso crecimiento económico de esta época influyó positivamente en las exportaciones que aumentaron notablemente gracias a diversas medidas fiscales y a la devaluación de la peseta. Así, el aumento de la demanda interna y externa favorecieron el crecimiento del tamaño medio empresarial y la mecanización progresiva de la fabricación, con el subsiguiente desplazamiento de la producción artesanal.

- Fase II. Entre mediados de los setenta y mitad de los noventa comenzaron a solicitarse productos de moda, modificando las condiciones de la demanda sujeta desde este momento a una rotación superior, con series más cortas, productos más exclusivos y de mayor calidad. Además, el sector protagonizó crisis periódicas como consecuencia de las reducciones de la demanda internacional, la crisis de precios de las materias primas y la competencia de precios entre los segmentos sobresalientes de la industria española. Como consecuencia de todo lo expuesto, se produjo la reducción del tamaño de las empresas y la subcontratación de parte de su producción, ya sea por razones estratégicas o de reducción de costes, dando lugar al desarrollo de la industria auxiliar. Es también en esta fase cuando se produjo la apertura al exterior de los mercados de la Unión Europea con el consecuente incremento de las importaciones de calzado asiático.

Fase III. Desde mitad de los noventa hasta la actualidad ha surgido un nuevo proceso de reestructuración condicionado por la aparición de la subcontratación de la fabricación y el descenso de los trabajadores en el sector. Este proceso ha fomentado la aparición de una economía informal en el sector y la deslocalización parcial de la producción. La demanda interior y exterior alcanza de nuevo niveles positivos, aunque la competencia internacional también sufre un aumento muy significativo, especialmente el calzado asiático. En este contexto la moda comienza a tener gran influencia por lo que el diseño, la calidad y la comercialización se convierten en las principales estrategias de diferenciación entre las producciones de los países líderes y los nuevos países productores. Asimismo, comienzan a emplearse nuevas tecnologías en el diseño, corte y logística. 
La globalización, la liberalización de los mercados y las nuevas tecnologías de la comunicación constituyen un nuevo contexto industrial, en el que los países emergentes como China o Brasil cuentan con una presencia cada vez más notable. Como consecuencia, el sector del calzado español y europeo se ha visto obligado a adaptarse a estas nuevas condiciones, sufriendo en este proceso una crisis sectorial que ha desembocado en una reducción de la producción. Por ello, en el intento de adaptarse al nuevo entorno han surgido empresas que importan y reexportan o importan exclusivamente, convivendo con las compañías tradicionales que se dedican a la fabricación de la totalidad o parte del producto. Así, se ha generado un sector internacionalizado en el que la marca, el diseño, la innovación y el control de la distribución y la logística suponen apoyos estratégicos para la actividad empresarial.

\subsection{Internacionalización del sector del calzado}

La industria española del calzado destaca por su carácter internacional, pues un gran número de empresas exportadoras destinan la mayor parte de su producción a los mercados exteriores (Miranda, 2014). El proceso de internacionalización de dichas empresas se inició de forma generalizada a finales de los años 60 . Este fue un proceso gradual, con un recorrido de muchas semejanzas con las etapas del modelo de Uppsala, especialmente en su versión actualizada, donde se destaca la importancia de las relaciones y las redes que establecen unas empresas con otras. En una primera fase, la estrategia de internacionalización se basó principalmente en la exportación y estuvo muy difundida en el sector. El crecimiento de las exportaciones españolas de calzado en los años 60 y la primera mitad de los 70 fue extraordinario. Su valor pasó de 124 millones de pesetas en 1960 a más de 7.600 millones en 1970 y más de 25.600 millones en 1975. En ambos períodos, el aumento de las exportaciones de la industria española fue mucho mayor en términos relativos que el crecimiento del comercio internacional de calzado y muy superior incluso al crecimiento de las exportaciones en Italia y Francia, que eran los países europeos que lideraban la exportación de calzado de cuero a principios del período.

La competitividad del calzado español residía fundamentalmente en su precio, que, comparando niveles de calidad semejantes, resultaba sensiblemente inferior al de sus competidores europeos (Roberts y Aw, 1986). De este modo, a pesar de la debilidad de la mayoría de las empresas productoras, de su desconocimiento inicial del mercado norteamericano y de la competencia de otros productores europeos con una posición más firme en este mercado, el calzado español pudo aprovechar su ventaja en precio para introducirse rápidamente en los Estados Unidos gracias a que fueron las propias compañías comercializadoras de calzado en este país las que se dirigieron a la industria española en busca de alternativas más baratas a las importaciones procedentes de Italia.

Aunque la mayor parte de las exportaciones eran realizadas por empresas de capital español, también hubo inversión directa norteamericana y europea, especialmente alemana, en la fabricación de calzado en España. De hecho, Miguel Hernández SA, una de las mayores empresas exportadoras de calzado a principios de los años 70, que se situó entre las cien primeras empresas del país por el valor de sus exportaciones, tenía el $99 \%$ de su capital en manos norteamericanas. En Elche, el principal centro de esta industria en el país, se crearon 22 empresas de calzado con inversión extranjera entre 1969 y 1974, el 13\% de las empresas del sector en la ciudad que registraron su constitución como sociedades en esos años, con una inversión extranjera que representó el $23 \%$ del capital total de dichas sociedades (Sevilla, 1985).

A partir de la década de 1990, un número de empresas reducido, comenzó a realizar inversiones directas en el exterior, con el objetivo de crear redes propias de comercialización y también poder establecer plantas de producción. A partir de esta época muchas empresas internacionalizaron su proceso productivo sin crear filiales en otros países, pues recurrieron a la subcontratación con firmas independientes. En esta década la industria no sólo profundizó en la descentralización empresarial y la especialización por fases del proceso productivo, sino que también mejoró su competitividad exterior elevando la calidad media del producto y cuidando más su diseño, e invirtiendo en la renovación tecnológica, la creación y promoción de marcas, la diversificación de los mercados y la formación de canales de distribución propios (Fuster, Martínez y Pardo, 2008). 
Algunas empresas dejaron de producir o subcontratar la producción en España y se transformaron en importadoras, tanto para abastecer el mercado español como para reexportar a otros países. La importación de calzado totalmente fabricado en Extremo Oriente y en otras zonas del mundo con bajos costes laborales, en ocasiones por encargo y con diseños de empresas españolas, alcanzó tal dimensión en España que en septiembre de 2009 se constituyó la Asociación de Importadores de Calzado y Complementos (ADICAL), la cual representa en la actualidad más de 150 marcas, con una facturación superior a los 367 millones de euros (Miranda, 2014). Otras empresas optaron por aumentar su competitividad desplazando a los países con menores costes solo parte de sus actividades productivas, aquellas más intensivas en trabajo no cualificado. En ocasiones se hizo mediante la constitución en el exterior de empresas filiales, pero más frecuentemente se trató de la subcontratación de estas tareas a empresas independientes en el extranjero y la sustitución de proveedores nacionales de componentes por proveedores de otros países (Díaz y González, 2006).

La deslocalización no es un fenómeno reciente en la industria, sino que ha formado parte de la estrategia de las grandes empresas multinacionales durante décadas. No obstante, su utilización se ha intensificado y generalizado a todo tipo de empresas desde los años 90, en numerosas actividades industriales y de servicios y en prácticamente todos los países desarrollados. Ello se ha debido tanto a la reducción de las barreras al comercio, que ha incrementado fuertemente la competitividad en el mercado mundial de los países con menores costes laborales (Krugman, 1995), como al progreso en las tecnologías de la información y la comunicación, que ha hecho posible dispersar internacionalmente las distintas fases de muchos procesos productivos (Hummels, Ishii y Yi, 2001).

\subsection{Ventajas de los distritos industriales}

Las empresas del calzado analizadas constituyen distritos industriales y, por ello, obtienen una serie de externalidades positivas derivadas de su localización. El origen de dichas externalidades como concepto económico se remonta a la obra de Alfred Marshall (1920). Este autor definió las economías de aglomeración como aquellas externalidades que se forman debido a la concentración espacial de empresas, identificando tres beneficios para las empresas derivados de la existencia de economías externas o de aglomeración. Estas son:

1. Acceso a un mercado de trabajo especializado que se genera como consecuencia de la aglomeración de empresas y que permite disponer de trabajadores altamente cualificados y especializados en las diferentes fases del proceso productivo que se realizan en el espacio de la aglomeración. Al mismo tiempo, el desarrollo de la aglomeración aumenta el interés de trabajadores externos a la misma, lo que redunda en un mejor ajuste entre oferta y demanda de trabajo.

2. Acceso a clientes y proveedores especializados, reduciendo de esta forma los costes de transacción y permitiendo, además, el intercambio fluido de información con proveedores y clientes sobre la mejora de sus productos y servicios.

3. Difusión del conocimiento e información sobre innovación y tecnología (los conocidos como desbordamientos de conocimiento o, en inglés, knowledge spillovers) entre empresas competidoras que se produce a través de contactos informales. Este intercambio de conocimiento, principalmente tácito, favorece una mejora de las capacidades competitivas y del nivel global de innovación de la aglomeración espacial de empresas.

También existen otros efectos positivos significativos como los menores costes de transporte, los retornos crecientes de escala o un mayor nivel de infraestructuras que mejoran a medida que nuevas empresas nacen dentro de la aglomeración o son atraídas hacia la misma con el objetivo de ser partícipes de dichos beneficios. Estos beneficios, como ya señaló Marshall, son externos a cada empresa, pero internos al territorio en el que se encuentran $y$, por tanto, específicos e intrínsecos al mismo.

La aglomeración espacial de empresas propicia así dos efectos principalmente. Por un lado, la dinámica de colocalización de nuevas empresas en el mismo territorio conduce a la generación de economías externas que, a su vez, se traducen en ventajas competitivas. Por otro lado, la proximidad geográfica de las empresas 
puede ser un elemento relevante para el desarrollo territorial de la propia aglomeración al generarse sinergias entre las empresas presentes en la misma (Filippi et al., 2015). Estos factores de proximidad dependen del crecimiento y desarrollo de la aglomeración espacial de empresas. Arthur (1990) estableció que los beneficios derivados de las economías de aglomeración se refuerzan conforme crece la propia aglomeración espacial de empresas. Como señalan Delgado, Porter y Stern (2014), las regiones que cuentan en sus espacios económicos con clústeres o distritos industriales tienden a mostrar mayores ratios de crecimiento y de innovación. Asimismo, dichas regiones también se muestran más atractivas ante empresas que buscan o bien relocalizarse o bien ubicar un nuevo establecimiento gracias a las condiciones favorables que ofrecen estas regiones y a la expectativa de obtener ventajas y/o beneficios derivados de la pertenencia o entrada a la aglomeración espacial de empresas (Litzel, 2017).

\subsection{Los distritos industriales como polos de atracción de inversión extranjera}

En el escenario económco actual, la globalización es una realidad incuestionable a la que se enfrentan las organizaciones. En este contexto, la internacionalización de las empresas se convierte prácticamente en una tarea necesaria para mantener su ventaja competitiva. Es por ello que cada vez más compañías deciden acceder a mercados exteriores para establecer sus sucursales (Dunning, 2009), siendo la industria del calzado una de las más internacionalizadas (Miranda, 2014).

Con la expansión internacional se persigue una gran variedad de objetivos; desde hacer frente a la madurez del mercado interno, mejorar la resistencia ante futuras crisis, obtener incentivos gubernamentales, la búsqueda de eficiencia, la proximidad entre proveedores y clientes o la diversificación del riesgo mundial, hasta la aplicación de sus recursos y capacidades en otros países o el desarrollo de nuevas competencias. El modelo OLI, también conocido como el paradigma ecléctico (Dunning, 1979), es el más utilizado por los académicos para tratar de comprender las inversiones extranjeras realizadas por las multinacionales. El modelo sostiene que las empresas deciden internacionalizarse si poseen una ventaja competitiva sobre el país anfitrión, distinguiendo tres tipos de ventajas competitivas diferentes: de propiedad, de localización y de internalización. Por consiguiente, las empresas acceden a mercados extranjeros si tienen activos específicos relevantes (ventajas de propiedad), pueden beneficiarse de la dotación de recursos y la posición geográfica del país de destino (ventajas de localización) y pueden organizar su propia producción en lugar de producir mediante acuerdos de cooperación (ventajas de internalización).

En relación con las ventajas de propiedad, diversos autores afirman que la internacionalización de las empresas que componen un país está estrechamente vinculada con la competitividad de dichas empresas (Porter, 1990; Kogut, 1991; Murtha y Lenway, 1994). De este hecho se derivan dos efectos: un "efecto empuje" que impulsa a las empresas ubicadas en un determinado país a buscar nuevas oportunidades en otras naciones dado el elevado nivel de competencia existente en el contexto nacional; y un "efecto arrastre" que atrae las inversiones extranjeras destinadas a aprovechar esa mayor competitividad derivada de las diferencias tecnológicas y organizativas entre países (Kogut, 1991).

Las beneficios precedentes de las economías de aglomeración, explicados en apartados anteriores, pueden mejorar la competitividad de las empresas y, por consiguiente, su proceso de internacionalización. En este sentido, tal y como señalan Hervas-Oliver y Boix-Domenech (2013), en la literatura sobre internacionalización existe la necesidad de reconocer las especificidades y las ventajas que se derivan de las aglomeraciones empresariales, puesto que la aglomeración empresarial es un factor clave que las multinacionales consideran cuando se ubican en un país extranjero (Jain, Kothari, y Kumar, 2016). Los distritos industriales, en particular, contienen una serie de recursos que las pequeñas empresas pueden aprovechar para iniciar y acelerar su internacionalización (Libaers y Meyer, 2011). Además, desde el enfoque opuesto, dichos entonos especializados han atraído continuamente a multinacionales de otros países con la esperanza de obtener ventajas derivadas de su localización (McCann y Mudambi, 2004), actuando como polos de atracción de la inversión extranjera, ya que el entorno de las empresas ubicadas en un distrito no sólo incluye a los agentes externos que forman parte de su cadena de valor, tales como proveedores, clientes o distribuidores (Mascarenhas, Baveja y Jamil, 1998; Schmid 
y Schurig, 2003), sino también instituciones apoyo, como universidades y centros de formación profesional (Landau, Karna, Richter y Uhlenbruck, 2016) que desempeñan un papel decisivo en la transferencia de conocimientos para el desarrollo de nuevas capacidades (Malmberg y Power, 2005; Knudsen, 2007; Tödling, Lehner y Kaufmann, 2009).

\section{Metodología}

La parte metodológica se ha divido en dos etapas bien diferenciadas entre sí, puesto que en cada una de ellas se emplean métodos de análisis diferentes. Las etapas planteadas para la realización de la presente investigación han sido: (1) Identificar los distritos industriales españoles del calzado localizados en las comarcas del Vinalopó y (2) analizar si dichos distritos industriales actúan como polos de atracción de la inversión extranjera.

\subsection{Etapa 1: identificación de los distritos industriales españoles del calzado de las comarcas del Vinalopó}

Para la identificación de los distritos industriales se ha seguido la metodología propuesta por el ISTAT (1996). Esta metodología ha sido utilizada por varios autores que se han ocupado del estudio de los distritos industriales en diferentes países. Para el caso español, Boix y Galleto (2004) la aplicaron para la elaboración, por primera vez, de un mapa de distritos industriales manufactureros. Es a partir de entonces, cuando han surgido varios trabajos en esta línea de investigación en diferentes sectores de actividad como, por ejemplo, la industria textil (PlaBarber y Puig, 2009) o el sector hotelero (Marco-Lajara et al., 2017). La metodología italiana utilizada por el ISTAT (1997) para la identificación de distritos industriales consta básicamente de dos etapas: la identificación de los Sistemas Locales de Trabajo (SLT) y la posterior verificación de éstos como Distritos Industriales (DI).

Por un lado, la primera de estas dos etapas consiste en la identificación de las principales áreas de concentración a nivel municipal. Se toma el municipio como unidad territorial básica de análisis debido a que los límites administrativos regionales son demasiado grandes para albergar el concepto de comunidad que postula la teoría del distrito. A partir de ahí, se trata de agrupar los municipios en sistemas locales de trabajo, en base a la población ocupada residente, la población ocupada total y los desplazamientos del lugar de trabajo de residencia al lugar de trabajo de la población. Por otro lado, la segunda etapa consiste en la verificación de que dichas áreas de concentración o sistemas locales de trabajo pueden ser considerados distritos industriales. De este modo, una vez han sido delimitados los SLT se procede al estudio de su estructura socioeconómica con el fin de identificar aquellos que pueden reunir los requisitos y, por tanto, ser considerados distritos industriales. Así, los SLT podrán ser considerados distritos industriales cuando el coeficiente de especialización sea superior a la unidad.

El coeficiente de especialización (CE) es una herramienta estadística que mide la presencia de una actividad en un territorio con respecto a la presencia de ese sector en toda la muestra de referencia. La variable utilizada, en este caso, para medir el grado de concentración de la actividad económica es el empleo y se expresa a través de la Figura 1.

Figura 1

Fórmula del coeficiente de especialización

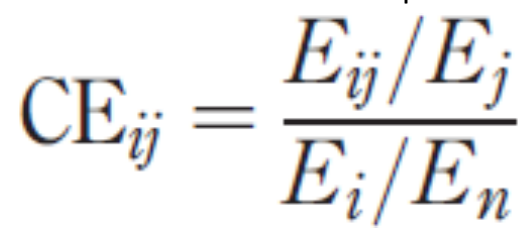

Fuente: Boix y Galleto (2004)

Donde Eij es el empleo en el sector i en la ubicación j, Ej es el empleo total en la ubicación j, Ei es el empleo en el sector i en todo el país, Et es el empleo total en el país. La interpretación de este coeficiente es la siguiente: cuando el valor del coeficiente es mayor que la unidad, esto indicaría que el empleo en una determinada industria 
y área geográfica es mayor que la media nacional, indicando justamente lo opuesto cuando el coeficiente adopta valores menores a la unidad. De este modo, a medida que este valor se aleja de la unidad, tanto en valores superiores como inferiores, indica la mayor/menor dependencia en términos de empleo que tiene un área sobre una determinada industria.

Los sistemas locales de trabajo españoles ya han sido previamente identificados por Boix y Galleto (2006a), cuyo trabajo sirve como base para realizar el presente análisis. En consecuencia, la tarea que se ha llevado a cabo consiste en comprobar con datos relativos al empleo en el sector del calzado del año 2020, cuáles de estos sistemas locales de trabajo siguen cumpliendo las características propias de un DI. Para comprobar los datos relativos al empleo en la industria del calzado del Vinalopó se han analizado un total de 16 municipios.

Con el propósito de ampliar el análisis, se ha utilizado otro coeficiente de especialización también ampliamente utilizado en los estudios empíricos (Malmberg y Lundequist, 2000; Kukalis, 2010; McCann y Folta, 2011). En este caso la concentración geográfica de la actividad manufacturera se mide a través de la densidad de empresas de la industria en cada área geográfica específica. Por lo tanto, la variable considerada ahora es el número de empresas en cada municipio y no el empleo en una determinada actividad (en el presente estudio la industria del calzado). En definitiva, el planteamiento que subyace en este coeficiente es el mismo que en el coeficiente anterior, pero cambia la variable empleada.

La población objeto de estudio la componen aquellos municipios de las comarcas del Vinalopó en los que se desarrollan actividades productivas propias de la industria del calzado. Respecto a la muestra, ésta está compuesta por 16 municipios, 14 de ellos pertenecientes a las comarcas del Vinalopó (Alto Vinalopó, Medio Vinalopó y Bajo Vinalopó), 1 municipio localizado en la comarca de la Vega Baja del Segura (también perteneciente a la provincia de Alicante) y otro municipio localizado en la región de Murcia. Finalmente, los 16 municipios fueron agrupados en 8 Distritos Industriales: Villena (D.1), Sax (D.2), Elda (D.3), Monóvar (D.4), Pinoso (D.5), Aspe (D.6), Crevillente (D.7) y Elche (D.8). A continuación, a través del Cuadro 1 se muestra el nombre de los 8 Distritos Industriales del calzado de las comarcas del Vinalopó y los municipios que conforman cada uno de los Sistemas Locales de Trabajo.

Cuadro 1

Distritos Industriales y Sistemas Locales de Trabajo del Vinalopó

\begin{tabular}{|c|c|}
\hline Distritos Industriales del Calzado & Sistemas Locales de Trabajo (SLT) \\
\hline Villena & Cañada (03052), Villena (03140) \\
\hline Sax & Salinas (03116), Sax (03123) \\
\hline Elda & Elda (03066), Petrer (03104) \\
\hline Monóvar & Monóvar (03089) \\
\hline Pinoso & $\begin{array}{c}\text { Algueña (03013), Pinoso (03105), Abanilla } \\
\text { (30001) }\end{array}$ \\
\hline Aspe & $\begin{array}{c}\text { Hondón de las Nieves (03077), Hondón de } \\
\text { los Frailes (03078), Aspe (03019) }\end{array}$ \\
\hline Crevillente & Crevillente (03059), San Isidro (03904 \\
\hline Elche & Elche (03065) \\
\hline
\end{tabular}

Fuente: Elaboración propia a partir de Boix y Trullén (2010)

Los datos utilizados para el análisis empírico llevado a cabo en la Etapa 1 provienen principalmente de fuentes externas secundarias (Camerdata y SABI). Por un lado, la base de datos Camerdata proporciona información sobre el empleo de la industria del calzado y del resto de actividades económicas en España, lo cual ha permitido calcular los coeficientes de aglomeración o especialización de la actividad económica, tanto a nivel de municipio como a nivel nacional. Cabe señalar que para la obtención de los datos se ha utilizado el código de la Clasificación Nacional de Actividades Económicas (CNAE) 15.2 'Fabricación de calzado', pues el análisis se centra en las empresas fabricantes de calzado. No obstante, dado que esta base de datos no ofrece información directamente 
sobre el número de empleados de cada empresa sino del rango de personal en el que se encuentra la empresa en cuestión, se procedió al cálculo del coeficiente de especialización tomando el punto medio de cada rango.

Por otro lado, de la base de datos $\mathrm{SABI}$ se ha obtenido información relativa a la localización de las empresas y su tamaño, lo cual ha permitido caracterizar cada uno de los 8 distritos industriales del calzado del Vinalopó en esta misma etapa de análisis. A continuación, se especifica la forma en que han sido medidas las variables utilizadas en el análisis:

- Grado de aglomeración

Como se ha explicado anteriormente, para medir el grado de aglomeración se ha empleado el Coeficiente de Especialización (CE) utilizando datos de empleo y número de empresas.

- Empleo

(Empleo en la industria del calzado en un determinado territorio/ Empleo total en ese mismo territorio) / (Empleo de la industria del calzado en el territorio nacional/ Empleo total nacional).

- Número de empresas

(Número de empresas de la industria del calzado en un determinado territorio/ número total de empresas en ese mismo territorio) / (Número de empresas de la industria del calzado en el territorio nacional/ Número total de empresas nacionales).

- Tamaño

El tamaño de las empresas ha sido medido a través del número de empleados y el criterio de dimensión utilizado ha sido el propuesto por la Comisión Europea, el cual distingue cuatro estratos de tamaño: Microempresa (menos de 10 trabajadores); PYME pequeña (entre 10 y 49 trabajadores); PYME mediana (entre 50 y 249 trabajadores) y gran empresa (más de 250 trabajadores).

\subsection{Etapa 2: los distritos zapateros del Vinalopó como polos de atracción de la inversión extranjera}

En el presente apartado se describe tanto la población como la muestra utilizada para el análisis empírico llevado a cabo en la Etapa 2. La población objeto de estudio de esta etapa de investigación está formada por todas las empresas dedicadas a la fabricación del calzado que se localizan en España y cuyo accionariado procede en su mayoría del extranjero. Así, los datos utilizados para el análisis empírico provienen principalmente de fuentes externas secundarias. Para analizar la procedencia del accionariado de las empresas del calzado instaladas en el Vinalopó se ha hecho uso de la base de datos $S A B I$ al poseer una vasta información sobre los temas requeridos. De este modo, a través de dicha base de datos se han seleccionado aquellas empresas con código CNAE 15.2 (Fabricación del calzado) cuyo accionariado procede, en su mayoría, del extranjero.

\section{Resultados}

A continuación, se muestran los resultados en dos etapas. La primera, trata de medir el nivel de especialización en la industria del calzado de los distritos españoles del calzado localizados en las comarcas del Vinalopó, así como analizar el tamaño de las empresas identificadas. Mientras que en la segunda etapa se analizan las empresas dedicadas a la fabricación del calzado localizadas en España que poseen capital procedente del extranjero.

\subsection{Resultados etapa 1}

A partir del procedimiento explicado en la parte de metodología, se han analizado un total de 16 municipios españoles pertenecientes a los 8 sistemas locales de trabajo especializados en el calzado en las regiones del 
Vinalopó. La composición de los diferentes distritos industriales identificados difiere en cuanto al número de municipios que forman cada uno de ellos, ya que mientras algunos distritos están formados por un solo municipio otros distritos engloban dos o más. El Cuadro 2 muestra los distritos identificados, la comarca en la que se encuentran localizadas, el número de empresas que alberga cada distrito, el número de empleados en la industria del calzado para cada distrito y los coeficientes de especialización productiva.

Respecto al coeficiente de especialización productiva basado en el empleo, los resultados muestran que en todos los casos este es mayor que la unidad. Por lo tanto, es posible afirmar que la proporción de empleo en el sector del calzado de las comarcas españolas del Vinalopó es superior a la media nacional. Sin embargo, el resultado de la aplicación de dicho coeficiente muestra diferentes grados de especialización. El valor del coeficiente varía entre 36,51 (Distrito Industrial de Aspe) y 95.41 (Distrito Industrial de Elda). En este último caso, la interpretación sería que el empleo generado en torno a la industria del calzado es 95 veces superior a la media nacional. De este modo, estas dos aglomeraciones representan los distritos industriales en los que el grado de especialización es menor y mayor, respectivamente. Así, entre estos dos extremos se encuentran los restantes distritos industriales con diferentes grados de especialización.

\section{Cuadro 2}

Distritos Industriales del calzado de las comarcas españolas del Vinalopó

\begin{tabular}{|c|c|c|c|c|c|c|}
\hline & Provincia & Comarca & $\begin{array}{c}\text { № } \\
\text { empresas }\end{array}$ & Empleo & $\begin{array}{c}\text { CE.1 } \\
\text { (Empleo) }\end{array}$ & $\begin{array}{c}\text { CE.2 } \\
\text { (Empresas) }\end{array}$ \\
\hline D.1 VILLENA & Alicante & $\begin{array}{c}\text { Alto } \\
\text { Vinalopó }\end{array}$ & 120 & 1.126 & 46,12 & 45,47 \\
\hline D.2 SAX & Alicante & $\begin{array}{c}\text { Alto } \\
\text { Vinalopó }\end{array}$ & 35 & 453 & 41,31 & 42,31 \\
\hline D.3 ELDA & Alicante & $\begin{array}{c}\text { Medio } \\
\text { Vinalopó }\end{array}$ & 522 & 6.024 & 95,41 & 75,30 \\
\hline D.4 MONOVAR & Alicante & $\begin{array}{c}\text { Medio } \\
\text { Vinalopó }\end{array}$ & 59 & 667 & 83,61 & 68,98 \\
\hline D.5 PINOSO & Alicante & $\begin{array}{c}\text { Medio } \\
\text { Vinalopó }\end{array}$ & 41 & 459 & 41,13 & 34,16 \\
\hline D.6 ASPE & Alicante & $\begin{array}{c}\text { Medio } \\
\text { Vinalopó }\end{array}$ & 60 & 550 & 36,51 & 33,53 \\
\hline D.7 CREVILLENTE & Alicante & $\begin{array}{c}\text { Bajo } \\
\text { Vinalopó }\end{array}$ & 118 & 1.187 & 40,05 & 42,85 \\
\hline D.8 ELCHE & Alicante & $\begin{array}{c}\text { Bajo } \\
\text { Vinalopó }\end{array}$ & 992 & 11.175 & 60,31 & 52,92 \\
\hline
\end{tabular}

Fuente: Elaboración propia.

Los resultados obtenidos al aplicar el coeficiente de especialización productiva basado en el número de empresas son bastante similares a los anteriores, puesto que todas las áreas especializadas superan la unidad. Además, los datos guardan una estrecha relación, ya que las regiones con mayor especialización en términos de empleo son también aquellas con mayor especialización en número de empresas. 
Gráfico 1

Coeficientes de especialización (CE) para la concentración de la industria del calzado del Vinalopó. Año 2020.

\section{0,00}

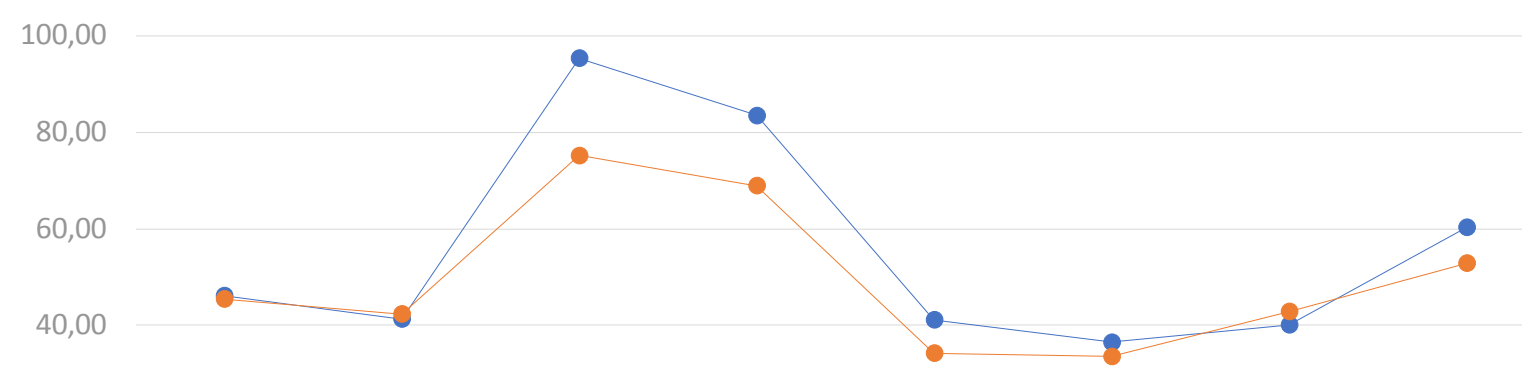

20,00

0,00

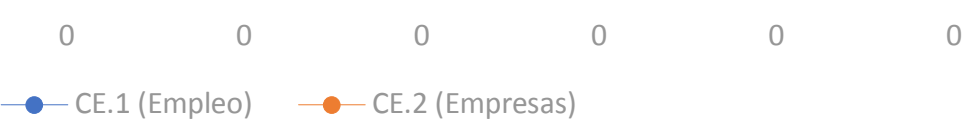

Fuente: Elaboración propia

El gráfico 1 permite analizar gráficamente las diferencias entre ambos coeficientes, mostrando los valores de cada coeficiente y distrito. Como es posible apreciar el CE basado en el empleo tiende a ser, de media, ligeramente superior a los valores obtenidos mediante el CE que utiliza el número de empresas como variable de medida. Ello puede deberse al efecto tamaño de las empresas, es decir, que el tamaño medio de las empresas ubicadas en un distrito sea superior/inferior al tamaño medio de las empresas ubicadas en otro y que, por lo tanto, se sobrevalore/infravalore el resultado obtenido en un coeficiente respecto al otro. En base a este razonamiento, parece más apropiado utilizar el coeficiente basado en el empleo, al menos cuando se analizan muestras de empresas heterogéneas en términos de tamaño

El Mapa 1 muestra la ubicación de los 8 distritos industriales del calzado identificados en las comarcas españolas del Vinalopó. Como se puede observar, existe una elevada cercanía entre distritos, pues todos ellos se encuentran localizados alrededor de un área de $400 \mathrm{Km}^{2}$, lo que pone de manifiesto la elevada especialización de las comarcas en la industria del calzado. Además, es posible señalar que dentro de las tres regiones (alto, medio y bajo Vinalopó), el medio Vinalopó, representado por el color azul, es la región que engloba más distritos (cuatro), repartiéndose los otros cuatro distritos a partes iguales entre las dos regiones restantes, las cuales se representan por el color naranja (el alto Vinalopó) y el color verde (el bajo Vinalopó).

Por lo que respecta al tamaño de las empresas que componen los distritos industriales indetificados es posible afirmar que de las 1.947 empresas existentes, el 71,19\% son microempresas, el 27,17\% son PYMES pequeñas, el $1,59 \%$ son PYMES medianas y únicamente el $0,05 \%$ son grandes empresas. Al considerar el tamaño de las empresas en cada uno de los distritos individualmente, lo cual es un indicador de la estructura productiva interna de cada uno de ellos, en general los resultados no varían respecto a los obtenidos de forma agregada. El Gráfico 2 muestran el tamaño de las empresas en los diferentes distritos identificados. 
Mapa 1.

Localización de los distritos industriales del calzado esapañoles de las comarcas del Vinalopó

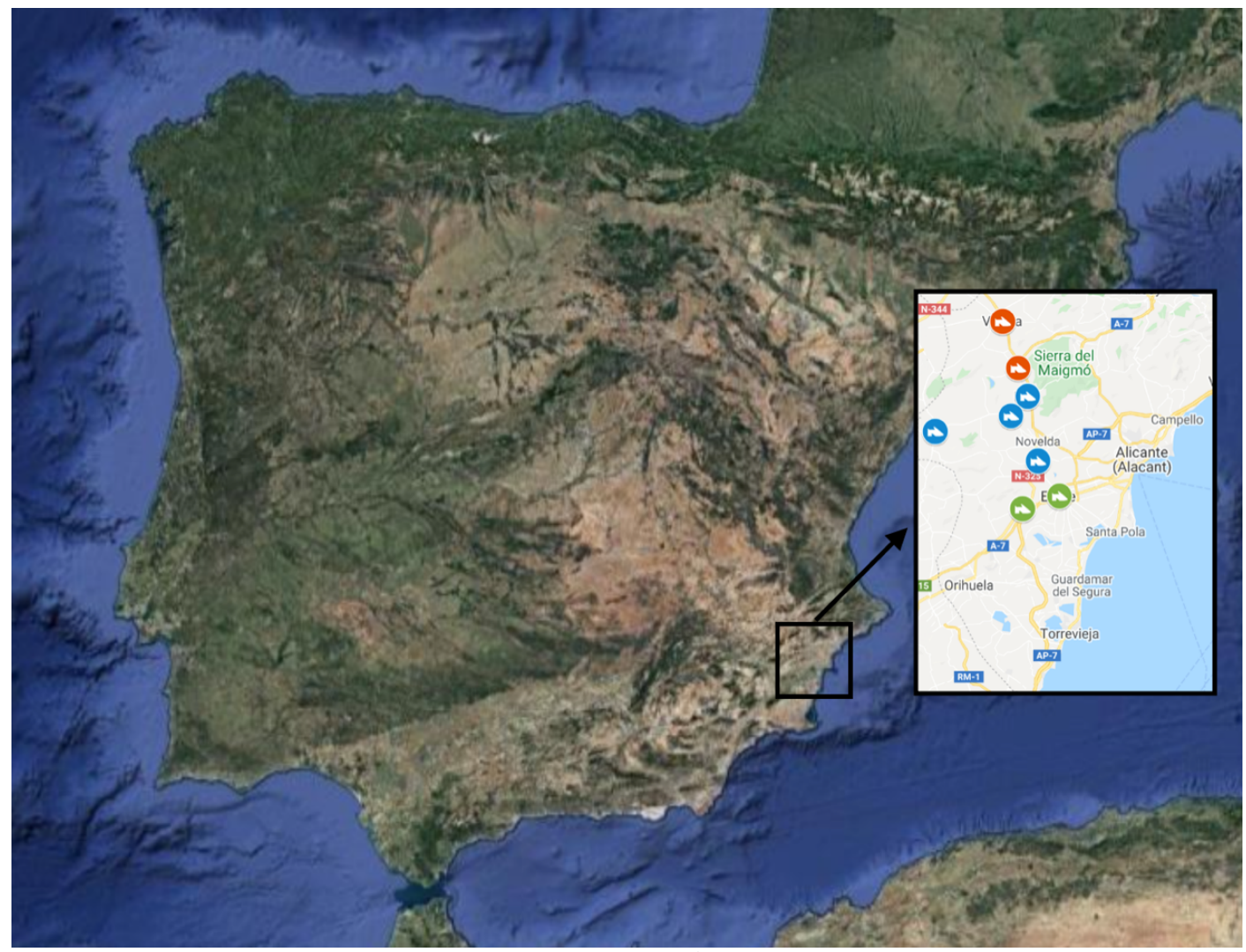

Fuente: Elaboración propia

Gráfico 2

Tamaño de las empresas en los distritos industriales del calzado del Vinalopó. Año 2020.

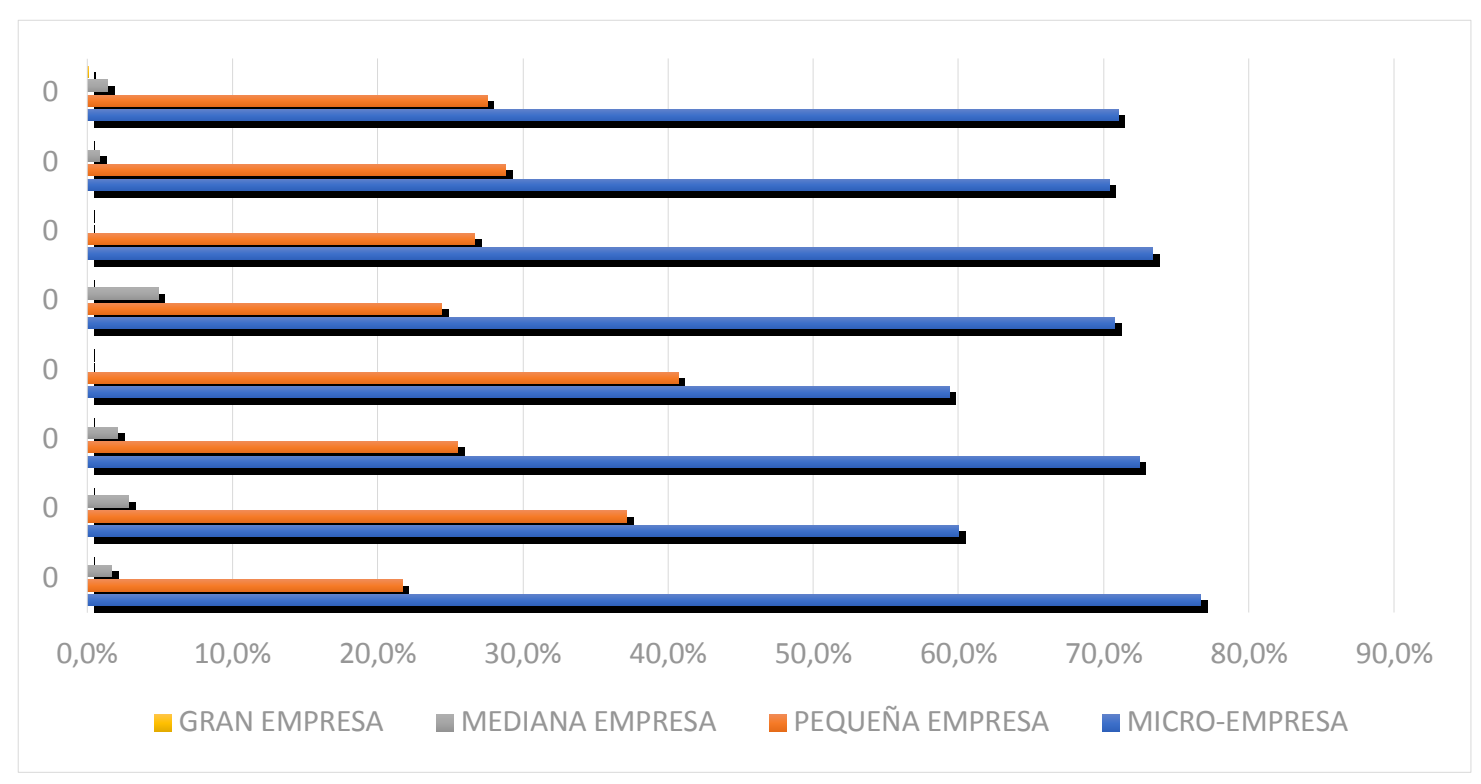

Fuente: Elaboración propia 
En cualquiera de los 8 distritos industriales del calzado analizados más del 95\% son microempresas y PYMES. De hecho, solamente un caso, el distrito de Elche, cuenta con una empresa grande. Esta es Pikolinos S.A. Por lo tanto, tomando este criterio de clasificación y a la luz de los resultados obtenidos, se constata la reducida dimensión de las empresas localizadas en los distritos. Así, tal y como sugiere la teoría del distrito industrial, estas áreas están especializadas en una industria y muestran una prevalencia por las pequeñas y medianas empresas.

\subsection{Resultados etapa 2}

Para poder analizar si las comarcas españolas del Vinalopó actúan como polos de atracción de la inversión extranjera se ha analizado el accionariado de todas las empresas en el territorio español dedicadas a la fabricación de calzado para conocer si verdaderamente el capital procede de España o, por el contrario, procede del extranjero.

Se han identificado 18 empresas con capital extranjero dedicadas a la fabricación del calzado en toda España, de las cuales 12 pertenecen a los distritos del Vinalopó ( 7 al distrito de Elda, 4 al distrito de Elche y 1 al distrito de Monóvar) ubicados en la provincia de Alicante. Las seis empresas restantes se localizan en La Rioja (Arnedo y Lardero), Albacete (Almansa), Madrid (San Sebastián de los Reyes), Barcelona (Castelldefels) y Valencia (Catarroja). Estos resultados guardan relación con las zonas donde existe un mayor desarrollo del calzado, pues Alicante, La Rioja y Albacete representan cerca del $80 \%$ de la producción de calzado a nivel nacional. Asimismo, en el Cuadro 3 se detallan los hallazgos de la investigación, pues se muestra en qué ciudad se encuentran ubicadas las empresas zapateras españolas con accionariado procedentes del exterior, así como el nombre, la ciudad y el país de procedencia de las empresas extranjeras con participación en estas últimas.

A la luz de los resultados, es posible afirmar que el $66,67 \%$ de la inversión extranjera en la industria de calzado español reside en las comarcas del Vinalopó, constatando la importancia del territorio en la industria zapatera. Asimismo, cabe resaltar el papel del capital estadounidense, pues el 50\% de las empresas zapateras extranjeras con inversión en España residen en Estados Unidos.

Cuadro 3

Inversión extranjera en las empresas fabricantes de calzado españolas. Año 2020

\begin{tabular}{|c|c|c|c|c|c|}
\hline $\begin{array}{c}\text { Empresa zapatera } \\
\text { española } \\
\text { participada }\end{array}$ & $\begin{array}{l}\text { Ciudad española } \\
\text { de procedencia }\end{array}$ & $\begin{array}{c}\text { Empresa extranjera } \\
\text { con participación en el } \\
\text { capital }\end{array}$ & $\begin{array}{l}\text { Ciudad de } \\
\text { procedencia de la } \\
\text { compañía con } \\
\text { participación }\end{array}$ & País & $\begin{array}{c}\% \text { de } \\
\text { participación }\end{array}$ \\
\hline Shoes By Stuart & Petrer & Tapestry INC & New York & $\begin{array}{l}\text { Estados } \\
\text { Unidos }\end{array}$ & $100 \%$ \\
\hline Creaciones SW S.A & Petrer & Tapestry INC & New York & $\begin{array}{l}\text { Estados } \\
\text { Unidos }\end{array}$ & $100 \%$ \\
\hline Shoe Heaven S.L & Petrer & Tapestry INC & New York & $\begin{array}{l}\text { Estados } \\
\text { Unidos }\end{array}$ & $100 \%$ \\
\hline Sunburst S.L & Petrer & Tapestry INC & New York & $\begin{array}{l}\text { Estados } \\
\text { Unidos }\end{array}$ & $100 \%$ \\
\hline Hope Diamon S.L & Petrer & Tapestry INC & New York & $\begin{array}{l}\text { Estados } \\
\text { Unidos }\end{array}$ & $100 \%$ \\
\hline Macaroni & Petrer & Tapestry INC & New York & $\begin{array}{l}\text { Estados } \\
\text { Unidos }\end{array}$ & $100 \%$ \\
\hline $\begin{array}{l}\text { Preparaciones y } \\
\text { Modeados S.L }\end{array}$ & Petrer & Tapestry INC & New York & $\begin{array}{l}\text { Estados } \\
\text { Unidos }\end{array}$ & $100 \%$ \\
\hline $\begin{array}{l}\text { New Millennium } \\
\text { Sports S.L }\end{array}$ & Elche & $\begin{array}{c}\text { Jinjiang Yuan Xiang } \\
\text { Garments Weaving } \\
\text { CO., LTD }\end{array}$ & Jinjiang & China & $80 \%$ \\
\hline $\begin{array}{l}\text { Move on Retail } \\
\text { Spain S.L }\end{array}$ & Elche & $\begin{array}{c}\text { Tata International } \\
\text { Limited }\end{array}$ & Mumbai & India & $100 \%$ \\
\hline
\end{tabular}




\begin{tabular}{|c|c|c|c|c|c|}
\hline Tan Shoes Spain & Elche & $\begin{array}{l}\text { Tanger International } \\
\text { Leather Company Til } \\
\text { Co Groupe }\end{array}$ & Tanger & Marruecos & $100 \%$ \\
\hline Sento's S.I & Elche & $\begin{array}{c}\text { Wpp Marketing } \\
\text { Communications Spain }\end{array}$ & London & $\begin{array}{c}\text { Gran } \\
\text { Bretaña }\end{array}$ & $68 \%$ \\
\hline Unisa Europa S.A & Monóvar & $\begin{array}{l}\text { Unisa Europa Holdings } \\
\text { INC }\end{array}$ & Miami & $\begin{array}{l}\text { Estados } \\
\text { Unidos }\end{array}$ & $100 \%$ \\
\hline Martino Forma S.L & Almansa & $\begin{array}{c}\text { California Shoe Group } \\
\text { INC }\end{array}$ & San Gabriel & $\begin{array}{l}\text { Estados } \\
\text { Unidos }\end{array}$ & $100 \%$ \\
\hline \multirow[t]{2}{*}{ Francisco Mendi } & \multirow[t]{2}{*}{ Lardero } & $\begin{array}{c}\text { Rahman Industries } \\
\text { Limited }\end{array}$ & \multirow[t]{2}{*}{$\begin{array}{l}\text { Val de Moder } \\
\text { Kanpur }\end{array}$} & Francia & $51 \%$ \\
\hline & & Superhouse LTD & & India & $51 \%$ \\
\hline $\begin{array}{c}\text { Calzados Rockport } \\
\text { S.L }\end{array}$ & $\begin{array}{c}\text { San Sebastian de } \\
\text { los Reyes }\end{array}$ & $\begin{array}{c}\text { Rockport Uk Holdings } \\
\text { LTD }\end{array}$ & London & $\begin{array}{l}\text { Reino } \\
\text { Unido }\end{array}$ & $100 \%$ \\
\hline $\begin{array}{l}\text { Linea de Seguridad } \\
\text { S.L }\end{array}$ & Castelldefels & Superhouse LTD & Kanpur & India & $100 \%$ \\
\hline Cipatex Europa S.L & Catarroja & $\begin{array}{l}\text { Cipatex administración } \\
\text { e participacoes LTDA. }\end{array}$ & Cerquilo & Brasil & $100 \%$ \\
\hline Sifac España S.L & Arnedo & $\begin{array}{c}\text { SIFAC - Sociedad } \\
\text { Industrial de Formas e } \\
\text { Artefacto }\end{array}$ & Oporto & Portugal & $100 \%$ \\
\hline
\end{tabular}

\section{Conclusiones}

En base a los resultados, es posible afirmar que los distritos industriales zapateros del Vinalopó identificados en trabajos previos (Boix y Galleto, 2004 y 2006a), siguen teniendo vigencia en la actualidad. Lo cual implica que, tras dos décadas después, estos territorios mantienen su especialización productiva en torno a la industria del calzado, superior a la media nacional. Asimismo, la aplicación de los coeficientes de especialización nos permite conocer aquellas distritos en los que existe una mayor dependencia a la industria del calzado. Así, los distritos de Elda y Monóvar son los que presentan una mayor dependencia, pues los dos coeficientes de especialización son superiores al resto de distritos analizados, lo que pone de manifiesta la reducida diversificación sectorial de dichos distritos. En el plano opuesto se encontrarían los distritos de Aspe y Crevillente, con una menor dependencia a la industria zapatera respecto al resto de distritos estudiados.

Por último, se han identificado un total de 18 empresas en España dedicadas a la fabricación de calzado con capital extranjero, de las cuales 12 pertenecen a los distritos del Vinalopó. Por lo tanto, es posible afirmar que el $66,67 \%$ de la inversión extranjera en la industria de calzado español reside en las comarcas del Vinalopó, constatando la importancia del territorio como polo de atracción de la inversión extranjera. De este modo, la investigación permite mostrar el efecto arrastre que ejercen los distritos zapateros del Vinalopó sobre el capital extranjero, así como la importancia del capital estadounidense en la industria del calzado, pues el 50\% de las empresas zapateras extranjeras con inversión en España residen en el país norteamericano.

\section{Referencias bibliográficas}

Arthur, W. B. (1990). Silicon Valley locational clusters: when do increasing returns imply monopoly? Mathematical Social Sciences, 19, 235-273.

Becattini, G. (1990). The Marshallian industrial district as a socioeconomic notion. En Pyke, F., Becattini, G. y Sengenberger, W. (Ed.), Industrial districts and interfirm co-operation in Italy (37-51). Geneva: International Institute for Labour Studies. 
Belso-Martinez, A. (2006). Do industrial districts influence export performance and export intensity? Evidence for Spanish SMEs' internationalization process. European Planning Studies, 14(6), 791-810.

Boix, R. y Galleto, V. (2006a). Sistemas locales de trabajo y distritos industriales marshallianos en España. Economía Industrial, 359, 165-184.

Boix, R. y Galletto, V. (2004). Identificación de sistemas locales de trabajo y distritos industriales en España. MITYC, Secretaría General de Industria, Dirección General de Política para la Pequeña y Mediana Empresa.

Boix, R. y Galletto, V. (2006b). El nuevo mapa de los distritos industriales de España y su comparación con Italia y el Reino Unido. Working Paper 06.04 Departament d'Economia aplicada, Universitat Autònoma de Barcelona.

Boix, R. y Trullén, J. (2010). Industrial districts, innovation and I-district effect: Territory or industrial specialization? European Planning Studies, 18(10), 1707-1729.

Cervera, M. (2003). Los clusters del calzado en el Vinalopó, Revista Valenciana de Economía y Hacienda, 8, 105187.

Climent, L. y Méndez, G. (2002). Medios innovadores y desarrollo local: los sistemas locales productores de calzado del interior de España. Estudios geográficos, 63(246), 5-31.

Delgado, M., Porter, M.E. y Stern, S. (2014). Clusters, convergence and economic performance. Research Policy, 43, 1785-1799.

Díaz, C. y González, B. (2006). Offshoring de producción: la creciente apuesta de las industrias manufactureras españolas, IX Encuentro de Economía Aplicada, Jaén, 8-10 de junio.

DIRCE. (2019). Directorio Central de Empresas. INE, Instituto Nacional de Estadística. España. Obtenido de : https://www.ine.es/prensa/dirce_2019.pdf

Dunning, J. (1979). Toward an eclectic theory of international production: Some empirical tests. Journal of International Business Studies, 11, 9-31.

Dunning, J. (2009). Location and the multinational enterprise: John Dunning's thoughts on receiving the JIBS 2008 decade award. Journal of International Business Studies, 40, 20-34.

Filippi, M., Triboulet, P., Chantelot, S. y Peres, S. (2015). The spatial distribution of French agricultural cooperatives: An exploratory spatial data analysis. European Planning Studies, 23(4), 710-732.

Fuster, B., Martínez, C. y Pardo, G. (2008). Las estrategias de competitividad de la industria del calzado ante la globalización. Revista de Estudios Regionales, 86, 71-96.

Hervas-Oliver, J. L. y Boix-Domenech, R. (2013). The economic geography of the meso-global spaces: Integrating multinationals and clusters at the localeglobal level. European Planning Studies, 21, 1064-1080.

Hummels, D., Ishii, J. y Yi, K. (2001). The nature and growth of vertical specialization in world trade. Joumal of International Economics, 54(1), 75-96.

Jain, N. K., Kothari, T. y Kumar, V. (2016). Location choice research: Proposing new agenda. Managing International Review, 56, 303-324.

Knudsen, M. P. (2007). The relative importance of interfirm relationships and knowledge transfer for new product development success. Journal of Product Innovation Management, 24(2), 117-138 
Kogut, B. (1991). Country capabilities and the permeability of borders. Strategic Management Journal, 12, 3347.

Krugman, P. (1995). Growing world trade: causes and consequences. Brooking Papers on Economic Activity, 1, 327-377.

Kukalis, S. (2010). Agglomeration economies and firm performance: The case of industry clusters. Journal of Management, 36(2), 453-481.

Landau, C., Karna, A., Richter, A. y Uhlenbruck, K. (2016). Institutional leverage capability: Creating and using institutional advantages for internationalization. Global Strategy Journal, 6, 50-68.

Libaers, D. y Meyer, M. (2011). Highly innovative small technology firms, industrial clusters and firm internationalization. Research Policy, 40, 1426-1437.

Litzel, N. (2017). Does embeddedness in clusters enhance firm survival and growth? An establishment-level analysis using CORIS data. Regional Studies, 51(4), 563-574.

Malmberg, A. y Power, D. (2005). (How) do (firms in) clusters create knowledge? Industry and Innovation, 12, 409-431.

Malmberg, A., Malmberg, B. y Lundequist, P. (2000). Agglomeration and firm performance: Economic of scale, localisation, and urbanisation among Swedish export firms. Environment and Planning A, 32(2), 305-321.

Marco-Lajara, B., Zaragoza-Sáez, P., Claver-Cortés, E., Úbeda-García, M. y García-Lillo, F. (2017). Tourist districts and internationalization of hotel firms. Tourism Management, 61, 451-464.

Marshall, A. (1920). Principles of Economics. London, UK: Macmillan.

Mascarenhas, B., Baveja, A. y Jamil, M. (1998). Dynamic of core competencies in leading multinational companies. California Management Review, 40, 117-132.

McCann, B. T. y Folta, T. B. (2011). Performance differentials within geographic clusters. Journal of Business Venturing, 26(1), 104-123.

McCann, P. y Mudambi, R. (2004). The location decision of the multinational enterprise: Some theoretical and empirical issues. Growth \& Change, 35, 491-524.

Miranda Encarnación, J. A. (2014). La internacionalización de la industria española del calzado: del auge exportador a la deslocalización, 1960-2013. Revista de la Historia de la Economía y de la Empresa, 8, 251283.

Murtha, T. y Lenway, S. (1994). Country capabilities and the strategic state: How national political institutions affect multinational corporations' strategies. Strategic Management Journal, 15, 113-129.

Pla-Barber, J. y Puig, F. (2009). Is the influence of the industrial district on international activities being eroded by globalization? Evidence from a traditional manufacturing industry. International Business Review, 18(5), 435-445.

Porter, M. (1990). The competitive advantage of nations. Harvard Business Review, 68, 73-94.

Roberts, M. J. y Aw, B. Y. (1986). Price and Quality Level Comparisons for US Footwear Imports: An Application of Multilateral Index Numbers. Pennsylvania University. 
Rosenthal, S. y Strange, W. C. (2001). The determinants of agglomeration. Journal of urban economics, 50(2), 191-229.

Schmid, S. y Schurig, A. (2003). The development of critical capabilities in foreign subsidiaries: Disentangling the role of the subsidiary's business network. International Business Review, 12, 755-782.

Sebastiá, R. A (1997). La industria del calzado en la provincia de Alicante: Características de su evolución reciente (1970-1991). Investigaciones Geográficas, 18, 81-98.

Sevilla, M. (1985). Crecimiento y urbanización. Elche 1960-1980. Universidad de Alicante-Ayuntamiento de Elche.

Tödling, F., Lehner, P. y Kaufmann, A. (2009). Do different types of innovation rely on specific kinds of knowledge interactions? Technovation, 29, 59-71.

Tortajada E., Fernández, L. e Ybarra-Pérez, J. (2005). Evolución de la industria española del calzado: factores relevantes en las últimas décadas. Economía Industrial, 355, 211-227.

Ybarra J.A. y Santamaría, M.J. (2005). El sector del calzado en España: retos ante un contexto de globalización. Boletín económico de ICE, Información Comercial Española, (2838), 9-23.

\footnotetext{
Esta obra está bajo una Licencia Creative Commons Attribución-NoCommercial 4.0 International

(cc) BY-NC
} 